\section{The Sequence and Integrated Analysis of Competing Endogenous RNAs Originating from Tea Leaves Infected by the Pathogen of Tea Leaf Spot, Didymella segeticola}

\author{
Yu Wang, ${ }^{1,2}$ Yuanyou Yang, ${ }^{1}$ Xinyue Jiang, ${ }^{1}$ Jiayan Shi, ${ }^{1}$ Yuqin Yang, ${ }^{1,3}$ Shilong Jiang, ${ }^{1,2}$ \\ Zhong $\mathrm{Li}^{2}{ }^{2}$ Delu Wang, ${ }^{4, \dagger}$ and Zhuo Chen ${ }^{1, \dagger}$ \\ ${ }^{1}$ Key Laboratory of Green Pesticide and Agricultural Bioengineering, Ministry of Education, Guizhou \\ University, Guiyang, Guizhou 550025, China \\ ${ }^{2}$ Agricultural College, Guizhou University, Guiyang, Guizhou 550025, China \\ ${ }^{3}$ College of Tea Science, Guizhou University, Guiyang, Guizhou 550025, China \\ ${ }^{4}$ College of Forestry, Guizhou University, Guiyang, Guizhou 550025, China
}

\begin{abstract}
Tea leaf spot, caused by Didymella segeticola, is an important disease which negatively affects the productivity and the quality of tea leaves. During infection by the pathogen, competing endogenous RNAs (ceRNAs) from tea leaves could contribute to achieving pathogenicity. In this study, circular RNAs (circRNAs) and long noncoding RNAs (IncRNAs), constituting ceRNAs, which share binding sites on microRNAs (miRNAs), and messenger RNAs (mRNAs) from infected and uninfected leaves of tea (Camellia sinensis 'Fuding-dabaicha') were sequenced and analyzed, and the identity and expression levels of the target genes of miRNA-mRNA and miRNA-IncRNA/circRNA were predicted. Analysis indicated that 10 mRNAs were bound by 20 miRNAs, 66 IncRNAs were bound by 40 miRNAs, and 17 circRNAs were bound by 29 miRNAs, respectively. For the regulation modes of ceRNAs, five ceRNA pairs were identified by the correlation analysis of IncRNA-miRNA-mRNA. For instance, expression of the xyloglucan endotransglycosylase gene in infected leaves was downregulated at the level of mRNA through miRNA PC-5p-3511474_3 binding with IncRNA TEA024202.1:MSTRG.37074.1. Gene annotation indicated that expression of this gene was significantly enriched in cell wall biogenesis and in the pathway of plant hormone signal transduction. The functional analysis of ceRNAs isolated from infected tea leaves will provide a valuable resource for future research on $D$. segeticola pathogenicity.
\end{abstract}

\section{ceRNA Announcement}

As a member of the Didymellaceae family, Didymella segeticola (Q. Chen) Q. Chen, Crous \& L. Cai was clustered in the clade of Didymella. It is known to be an important phytopathogen, causing various crop diseases (Chen et al. 2015, 2017) such as leaf spot on Tibetan thistle (Chen et al. 2015), tea (Camellia sinensis) (Zhao et al. 2018; Ren et al. 2019), and tobacco (Guo et al. 2020). Tea leaf spot caused by $D$. segeticola is a novel foliar disease of tea first discovered in the past 3 years, which prefers to occur in the cold spell period in late spring or in tea plantations at higher altitude (Ren et al. 2019; Zhao et al. 2018). Due to the lack of effective control measures and our limited understanding of its epidemiology, tea leaf spot disease often causes huge losses in the production of tea leaves, as well as a

${ }^{\dagger}$ Corresponding authors: D. L. Wang; dlwang@gzu.edu.cn, and Z. Chen; gychenzhuo@aliyun.com

*The $e$-Xtra logo stands for "electronic extra" and indicates that a supplementary file is published online.

The author(s) declare no conflict of interest.

Accepted for publication 24 August 2021.

C 2022 The American Phytopathological Society
Funding

This research was funded by National Key Research Development Program of China (2017YFD0200308) and its postsubsidy project ([2018]5262), the National Natural Science Foundation of China (21977023 and 31860515), and the Program of Introducing Talents to Chinese Universities (111 Program, D20023).

\section{Keywords}

competing endogenous RNA, Didymella segeticola, tea leaf spot 
decrease in the quality of the tea leaves (Zhao et al. 2018). Although we have screened some biofungicides for activity against $D$. segeticola and studied the mode of action of microbial biofungicides such as phenazine-1-carboxylic acid, Ningnanmycin, and Zhongshengmycin, the lack of knowledge about the pathogenicity of this fungal pathogen and the epidemic rule of the disease limits the effectiveness of any control technology in the field (Ren et al. 2021; Yang et al. 2021b; Yin et al. 2021).

The messenger RNA (mRNA) interaction with other RNAs allows for posttranscriptional control of gene expression by interfering with mRNA translation and stability and is involved in the control of biological processes such as growth, development, and environmental responses of living organisms (Hansen et al. 2013; Tay et al. 2014). In our previous study, the mRNAs and microRNAs (miRNAs) of $D$. segeticola and the tea host were sequenced, and the candidate target genes of the miRNA in the pathogen and the tea host were identified. The results indicated that the mechanism and network of mRNA-miRNA interactions between the pathogen and tea host were complex (Yang et al. 2021a). Competing endogenous RNAs (ceRNAs) are endogenous RNA transcripts, which include mRNAs, circular RNAs (circRNAs), and long noncoding RNAs (IncRNAs), and are mutual miRNA response elements, regulating the expression of different RNA species by competing for the same binding sites in miRNAs (Hansen et al. 2013; Tay et al. 2014). Therefore, ceRNAs of a plant host could function as disease resistance determinants or pathogenicity inhibitors against infection by a phytopathogen at the level of IncRNA, circRNA, and so on (Cui et al. 2020). Therefore, we hypothesized that ceRNAs could be used to provide insights into the pathogenicity of $D$. segeticola in the tea host.

In this study, single conidia of $D$. segeticola strain CGMCC3.20152 were incubated on potato dextrose agar (PDA) at $28^{\circ} \mathrm{C}$ in the dark for 10 days to obtain single-spore colonies for the pathogenicity assay and ceRNA analysis. Five-year-old tea (C. sinensis 'Fudingdabaicha') bushes were grown in the greenhouse, being maintained at $25^{\circ} \mathrm{C}$ during the day and $20^{\circ} \mathrm{C}$ at night, with cycles of $14 \mathrm{~h}$ of light and $10 \mathrm{~h}$ of darkness and a relative humidity of 70 to $80 \%$. Healthy tea twigs at the one-bud and fifth-leaf stages were cut from the tea bushes (Li et al. 2019). Tea leaves were punctured with a sterile needle to facilitate infection, then inoculated with $D$ segeticola mycelial plugs on infected leaves or sterile PDA plugs on uninfected leaves. After inoculation for $48 \mathrm{~h}$, a lesion formed rapidly on each leaf inoculated with mycelial plugs but not on leaves inoculated with sterile PDA plugs. Each treatment was carried out with three biological replicates.

The total RNA of tea leaves was extracted using TRIzol reagent (Invitrogen, Carlsbad, CA, U.S.A.), following the manufacturer's instructions. The amount and purity of each RNA sample were quantified using a NanoDrop ND-1000 spectrophotometer (Thermo Fisher, Wilmington, DE, U.S.A.). RNA integrity (RIN) was assessed by the Agilent 2100 Bioanalyzer (Agilent Technologies, Palo Alto, CA, U.S.A.), with a suitable RIN number needing to be $>7.0$. The complementary DNA (cDNA) libraries were prepared using TruSeq Stranded Total RNA HT Sample Prep Kit (Illumina, San Diego, CA, U.S.A.) and TruSeq Small RNA Sample Prep Kits (Illumina). The mRNAs, IncRNAs, circRNAs, and miRNAs were sequenced using the cDNA libraries on an Illumina HiSeq 4000 at Lc-bio Technologies Co., Ltd. (Hangzhou, China), following the manufacturer's recommended protocol. Cutadapt (Martin 2011) and in-house Perl script were used to remove low-quality reads, including adapters, low-quality bases, and undetermined bases. Then, the sequence quality was verified using FastQC (https://www. bioinformatics.babraham.ac.uk/projects/fastqc/) to obtain clean reads for subsequent downstream analyses.

For mRNAs, clean reads were aligned to the reference genome of $C$. sinensis var. sinensis using HISAT software (version 2.0.4) (http://tpdb.shengxin.ren/index.html) (Kim et al. 2015). The mapped reads of each sample were assembled; then, the expression abundance was quantified by calculating the value of fragments per kilobase of transcript per million mapped reads, with differentially expressed transcripts from infected leaves, relative to uninfected leaves, being identified at the level of $\log _{2}$ (fold change) $>1$ or $\log _{2}$ (fold change) $<-1$ and with statistical significance $(P$ value $<0.05)$, using StringTie software (version stringtie-1.3. 4d) (https://ccb.jhu.edu/software/stringtie/) (Frazee et al. 2015; Pertea et al. 2015) and the Ballgown package in $\mathrm{R}$ (Frazee et al. 2015).

For IncRNAs, sequence quality was further verified using FastQC (https://www.bioinformatics. babraham.ac.uk/projects/fastqc/). Valid reads were used to map reads to the genome of $C$. sinensis var. sinensis using Bowtie2 in R (Langmead and Salzberg 2012) and TopHat2 (http://ccb. jhu.edu/software/tophat) (Kim et al. 2013). The mapped reads of each sample were assembled 
using StringTie software (version stringtie-1.3.4d) (https://ccb.jhu.edu/software/stringtie/) (Pertea et al. 2015); then, the transcriptomes from the six samples (three replicates of each of the infected and uninfected treatments) were merged to construct a comprehensive transcriptome using the Perl program (https://www.perl.org). After the final transcriptome was generated, the abundance of each of the transcripts was estimated using StringTie software (Pertea et al. 2015) and the Ballgown package in R (Frazee et al. 2015). All transcripts were filtered according to the previously described methods (Li et al. 2021). After all transcripts with a coding potential calculator $(\mathrm{CPC})$ score $<-1$ and the coding/noncoding index $(\mathrm{CNCl})$ score $<0$ were removed, the remaining transcripts with coding potential were used for prediction, using CPC software (version 0.9-r2) (http://cpc2.gao-lab.org/) (Kong et al. 2007) and CNCl software (version 2.0) (https:// github.com/www-bioinfo-org/CNCl) (Sun et al. 2013). The remaining transcripts were considered to be IncRNAs. The expression level of IncRNAs was calculated using StringTie software (Pertea et al. 2015; Trapnell et al. 2010) and Ballgown package in R (Frazee et al. 2015).

For circRNAs, Bowtie2 (version 2.0.0-beta4) and TopHat2 software (http://ccb.jhu.edu/ software/tophat) were used to map reads to the tea genome (Kim et al. 2013; Langmead and Salzberg 2012). The remaining, unmapped reads were still mapped to the genome, using TopHat-Fusion software (http://tophat-fusion.sourceforge.net) (Kim and Salzberg 2011). CIRCExplorer 2 software (https://github.com/YangLab/CIRCexplorer2) was used to assemble the mapped reads to circRNAs de novo (Zhang et al. 2014, 2016). All samples generated unique circRNAs. The differentially expressed circRNAs, from infected relative to uninfected leaves, were selected with $\log _{2}$ (fold change) $>1$ or $\log _{2}$ (fold change) $<-1$ and with statistical significance $(P$ value $<0.05)$ by $\mathrm{R}$ package-empirical analysis of differentially expressed genes in R (edgeR) (version 3.13) (http://bioconductor.org/packages/release/bioc/html/edgeR. html) (Robinson et al. 2010).

For miRNAs, raw reads were analyzed using ACGT101-miR (LC Sciences, Houston, TX, U.S.A.) to remove adapter dimers and low-complexity sequences, and were filtered by aligning the databases of mRNA, RFam (containing ribosomal RNA, transfer RNA, small nuclear RNA, and small nucleolar RNA) (http://rfam.janelia.org), and the repetitive DNA element database Repbase (https://www.girinst.org/repbase). Reads with lengths of 18 to 25 nucleotides (nt) (i.e., miRNA-like) were retained, and unique sequences with lengths in the range 18 to $25 \mathrm{nt}$ were mapped to species-specific precursors in miRBase 22.0 (ftp://mirbase.org/pub/mirbase/CURRENT/) by BLAST search, to identify known miRNAs and novel $3 p$ - and $5 p$-derived miRNAs. The rest of the sequences were aligned through the databases of mRNA, RFam, and Repbase and filtered to form valid reads.

The software used for the miRNA-mRNA part was TargetFinder (https://github.com/ carringtonlab/TargetFinder) (Allen et al. 2005; Manke et al. 2008; Mrowka et al. 2008; Wasserman and Sandelin 2004). The part of miRNAs-IncRNAs/circRNAs was conducted using the ssearch36 program (36.3.6), based on the Smith-Waterman algorithm (Farrar 2007; Pearson 2016; Smith and Waterman 1981).

In all, 20, 89, and 36 of the ceRNA interaction pairs were predicted in the miRNAmRNA, miRNA-IncRNA, and miRNA-IncRNA interactions, respectively. The number of miRNA-IncRNA interactions was significantly higher than that of either miRNA-mRNA or miRNA-circRNA. For analysis of the interaction pairs in IncRNA/circRNA-miRNA-mRNA, five pairs of ceRNAs were found, with the regulation mode being divided into two types; namely, circRNA-miRNA-mRNA (type 1) and IncRNA-miRNA-mRNA (type 2). For type 1, no ceRNA pairs existed in the regulation mode. For type 2, five pairs of ceRNAs of 5 IncRNAs/4 miRNAs/4 mRNAs were predicted. Among the five pairs, IncRNA TEA010600.1:MSTRG.70981.3 bound miRNA PC-5p-129222_31. Meanwhile, when IncRNA TEA010600.1:MSTRG.70981.3 was upregulated, expression of the PROTEIN NETWORKED 1D gene, targeted by miRNA PC-5p129222_31, was also significantly upregulated. IncRNA TEA024202.1:MSTRG.37074.1 bound to miRNA of PC-5p-3511474_3. When IncRNA TEA024202.1:MSTRG.37074.1 was downregulated, expression of the xyloglucan endotransglycosylase (XET) gene targeted by PC-5p-3511474_3 was significantly downregulated. In addition, IncRNAs .:MSTRG.38793.2, .:MSTRG.44844.1, and :MSTRG.67275.1 bound miRNAs PC-5p-3941079_3, PC-5p-4527030_2, and PC-5p-4527030_2, respectively. When these three IncRNAs were significantly up- or downregulated, expression of all genes targeted by the corresponding miRNAs was also significantly, reversibly regulated.

The genes regulated by the differentially expressed ceRNAs were annotated using the Gene Ontology database, and the total number of genes (TB gene numbers) and number of genes with significantly different expression between infected and uninfected treatment 
Table 1. Competing endogenous RNA (ceRNA) sequences from tea (Camellia sinensis) leaves during infection by the fungal pathogen Didymella segeticola

\begin{tabular}{|c|c|c|c|c|}
\hline \multirow[b]{2}{*}{ RNAs $^{a}$} & \multirow[b]{2}{*}{ Sequence } & \multicolumn{2}{|c|}{ Treatment $^{\mathrm{b}}$} & \multirow[b]{2}{*}{ Results of correlation analysis } \\
\hline & & Uninfected & Infected & \\
\hline \multicolumn{5}{|l|}{ Raw data } \\
\hline \multirow[t]{3}{*}{ miRNA } & Raw reads & $16,673,700$ & $13,651,746$ & - \\
\hline & Valid reads & $13,335,514$ & $10,804,656$ & - \\
\hline & Valid bases & - & - & - \\
\hline \multirow[t]{3}{*}{ IncRNA/circRNA } & Raw reads & $141,647,262$ & $135,085,399$ & - \\
\hline & Valid reads & $139,330,285$ & $132,648,511$ & - \\
\hline & Valid bases & $20.9 \mathrm{G}$ & $19.89 \mathrm{G}$ & - \\
\hline \multirow[t]{3}{*}{ mRNA } & Raw reads & $46,410,408$ & $47,926,218$ & - \\
\hline & Valid reads & $45,522,197$ & $47,071,604$ & - \\
\hline & Valid bases & $6.83 \mathrm{G}$ & $7.06 \mathrm{G}$ & - \\
\hline \multicolumn{5}{|l|}{ Regulation mode of ceRNAs } \\
\hline miRNA-mRNA & - & - & - & 20 pairs, 10 mRNAs/18 miRNAs \\
\hline miRNA-IncRNA & - & - & - & 89 pairs, 66 IncRNAs/40 miRNAs \\
\hline miRNA-circRNA & - & - & - & 36 pairs, 29 circRNAs/17 miRNAs \\
\hline IncRNA/circRNA-miRNA-mRNA & - & - & - & 5 pairs, 5 IncRNAs/0 circRNA/4 miRNAs/4 mRNAs \\
\hline Type 1: circRNA-miRNA-mRNA & - & - & - & 0 pair, 0 circRNA/0 miRNA/0 mRNA \\
\hline Type 2: IncRNA-miRNA-mRNA & - & - & - & 5 pairs, 5 IncRNAs/4 miRNAs/4 mRNAs \\
\hline
\end{tabular}

samples (TS gene numbers) were 12,931 and 3, respectively. At the level of biological process, expression of the XET gene, which was regulated by the differentially expressed ceRNAs, was enriched with respect to cell wall biogenesis. At the level of cellular component, expression of the XET gene was enriched with respect to apoplast and cell wall whereas, at the level of molecular function, the XET gene was enriched with respect to hydrolyzing O-glycosyl compounds. When the Kyoto Encyclopedia of Genes and Genomes database annotation of the genes regulated by the differentially expressed ceRNAs was carried out, TB and TS gene numbers were 15,776 and 3, respectively. Expression of the XET gene regulated by the differentially expressed ceRNAs was enriched in the pathway of plant hormone signal transduction (Table 1).

For tea leaf spot caused by $D$. segeticola, ceRNAs, including mRNAs, IncRNAs, circRNAs, and miRNAs from $D$. segeticola-infected and uninfected tea leaves, were sequenced and analyzed. This dataset will provide an important resource for studying the pathogenicity of the pathogen in the tea host. The sequences of mRNAs, IncRNAs, circRNAs, and miRNAs of tea during infection by $D$. segeticola have been deposited in GenBank under Sequence Read Archive accession number PRJNA528172 for mRNAs, PRJNA689791 for IncRNAs and circRNAs, and PRJNA534364 for miRNAs. In addition, bioinformatics analysis of ceRNAs is presented in Supplementary Table S1. The sequences and functional analysis of ceRNAs and miRNAs originating from infected tea leaves will provide a valuable resource for future research on trait-specific genes of the pathogen, host-pathogen interactions, and disease resistance in the tea host.

\section{Literature Cited}

Allen, E., Xie, Z., Gustafson, A. M., and Carrington, J. C. 2005. microRNAdirected phasing during trans-acting siRNA biogenesis in plants. Cell 121: 207-221.

Chen, Q., Hou, L.-W., Duan, W.-J., Crous, P.-W., and Cai, L. 2017. Didymellaceae revisited. Stud. Mycol. 87:105-159.

Chen, Q., Zhang, K., Zhang, G.-Z., and Cai, L. 2015. A polyphasic approach to characterise two novel species of Phoma (Didymellaceae) from China. Phytotaxa 197:267-281.
Cui, J., Jiang, N., Hou, X.-X., Wu, S.-H., Zhang, Q., Meng, J., and Luan, Y.-S. 2020. Genome-wide identification of IncRNAs and analysis of ceRNA networks during tomato resistance to Phytophthora infestans. Phytopathology 110:456-464.

Farrar, M. 2007. Striped Smith-Waterman speeds database searches six times over other SIMD implementations. Bioinformatics 23:156-161.

Frazee, A. C., Pertea, G., Jaffe, A. E., Langmead, B., Salzberg, S. L., and Leek, J. T. 2015. Ballgown bridges the gap between transcriptome assembly and expression analysis. Nat. Biotechnol. 33:243-246. 
Guo, Z.-N., Xie, H.-L., Wang, H.-C., Huang, Y., Chen, Q.-L., Xiang, L.-G., and Yang, X.-H. 2020. Leaf spot caused by Didymella segeticola on tobacco in China. Plant Dis. 104:1559.

Hansen, T. B., Jensen, T. I., Clausen, B. H., Bramsen, J. B., Finsen, B., Damgaard, C. K., and Kjems, J. 2013. Natural RNA circles function as efficient microRNA sponges. Nature 495:384-388.

Kim, D., Langmead, B., and Salzberg, S. L. 2015. HISAT: A fast spliced aligner with low memory requirements. Nat. Methods 12:357-360.

Kim, D., Pertea, G., Trapnell, C., Pimentel, H., Kelley, R., and Salzberg, S. L. 2013. TopHat2: Accurate alignment of transcriptomes in the presence of insertions, deletions and gene fusions. Genome Biol. 14:R36.

Kim, D., and Salzberg, S. L. 2011. TopHat-fusion: An algorithm for discovery of novel fusion transcripts. Genome Biol. 12:R72.

Kong, L., Zhang, Y., Ye, Z.-Q., Liu, X.-Q., Zhao, S.-Q., Wei, L., and Gao, G. 2007. CPC: Assess the protein-coding potential of transcripts using sequence features and support vector machine. Nucleic Acids Res. 35:W345-W349.

Langmead, B., and Salzberg, S. L. 2012. Fast gapped-read alignment with Bowtie 2. Nat. Methods 9:357-359.

Li, D.-X., Bao, X.-T., Ren, Y.-F., Wang, Y., Song, B.-A., and Chen, Z. 2019. First report of Lasiodiplodia theobromae causing leaf spot on tea plant in Guizhou Province of China. Plant Dis. 103:374.

Li, D.-X., Jiang, S.-L., Wen, X.-D., Song, X.-C., Yang, Y.-Y., Wang, Y., Huang, H.-L., Wang, D.-L., and Chen, Z. 2021. Sequencing and functional annotation of mRNAs and IncRNAs from tea (Camellia sinensis) leaves during infection by the fungal pathogen, Lasiodiplodia theobromae. PhytoFrontiers. 1:364-367.

Manke, T., Roider, H. G., and Vingron, M. 2008. Statistical modeling of transcription factor binding affinities predicts regulatory interactions. PLOS Comput. Biol. 4:e1000039.

Martin, M. 2011. Cutadapt removes adapter sequences from high-throughput sequencing reads. EMBnet. J. 17:10-12.

Mrowka, R., Blüthgen, N., and Fähling, M. 2008. Seed-based systematic discovery of specific transcription factor target genes. FEBS J. 275:3178-3192.

Pearson, W. R. 2016. Finding protein and nucleotide similarities with FASTA. Curr. Protoc. Bioinf. 53:3.9.1-3.9.25.

Pertea, M., Pertea, G. M., Antonescu, C. M., Chang, T. C., Mendell, J. T., and Salzberg, S. L. 2015. StringTie enables improved reconstruction of a transcriptome from RNA-seq reads. Nat. Biotechnol. 33:290-295.

Ren, Y.-F., Li, D.-X., Jiang, S.-L., Wang, Y., Tang, Q., Huang, H.-L., Wang, D.-L., Song, B.-A., and Chen, Z. 2021. Integration of transcriptomic and proteomic data reveals the possible action mechanism of the antimicrobial Zhongshengmycin against Didymella segeticola, the causal agent of tea leaf spot. Phytopathology 111:2238-2249.
Ren, Y.-F., Li, D.-X., Zhao, X.-Z., Wang, Y., Bao, X.-T., Wang, X., Wu, X., Wang, D.-L., Song, B.-A., and Chen, Z. 2019. Whole genome sequences of the tea leaf spot pathogen Didymella segeticola. Phytopathology 109:1676-1678.

Robinson, M. D., McCarthy, D. J., and Smyth, G. K. 2010. Edger: A bioconductor package for differential expression analysis of digital gene expression data. Bioinformatics 26:139-140.

Smith, T. F., and Waterman, M. S. 1981. Identification of common molecular subsequences. J. Mol. Biol. 147:195-197.

Sun, L., Luo, H.-T., Bu, D.-C., Zhao, G.-G., Yu, K.-T., Zhang, C.-H., Liu, Y.-N., Chen, R.-S., and Zhao, Y. 2013. Utilizing sequence intrinsic composition to classify protein-coding and long non-coding transcripts. Nucleic Acids Res. 41:e166.

Tay, Y., Rinn, J., and Pandolfi, P. P. 2014. The multilayered complexity of ceRNA crosstalk and competition. Nature 505:344-352.

Trapnell, C., Williams, B. A., Pertea, G., Mortazavi, A., Kwan, G., Van Baren, M. J., Salzberg, S. L., Wold, B. J., and Pachter, L. 2010. Transcript assembly and quantification by RNA-seq reveals unannotated transcripts and isoform switching during cell differentiation. Nat. Biotechnol. 28:511-515.

Wasserman, W. W., and Sandelin, A. 2004. Applied bioinformatics for the identification of regulatory elements. Nat. Rev. Genet. 5:276-287.

Yang, R., Jiang, S.-L., Li, D.-X., Yin, Q.-X., Wu, X., Wang, Y., Wang, D.-L., and Chen, Z. 2021a. Integrated mRNA and small RNA sequencing for analyzing leaf spot pathogen Didymella segeticola and its host, tea (Camellia sinensis), during infection. Mol. Plant-Microbe Interact. 34:127-130.

Yang, R., Jiang, S.-L., Wen, X.-D., Song, X.-C., Wang, X., Li, D.-X., Yin, Q.-X., Wu, X., Wang, D.-L., and Chen, Z. 2021b. The antifungal activity and mode of action of a Streptomyces-derived anti-microbial, Ningnanmycin, on the tea gray blight disease pathogen Pseudopestalotiopsis camelliae-sinensis. Phytopathology 111:1735-1742.

Yin, Q.-X., Yang, R., Ren, Y.-F., Yang, Z.-Y., Li, T., Huang, H.-L., Tang, Q., Li, D.-X., Jiang, S.-L., Wu, X., Wang, D.-L., and Chen, Z. 2021. Transcriptomic, biochemical and morphological study reveals the mechanism of inhibition of Pseudopestalotiopsis camelliae-sinensis by Phenazine-1-carboxylic acid. Front. Microbiol. 12:468.

Zhang, X.-O., Dong, R., Zhang, Y., Zhang, J.-L., Luo, Z., Zhang, J., Chen, L.-L., and Yang, L. 2016. Diverse alternative back-splicing and alternative splicing landscape of circular RNAs. Genome Res. 26:1277-1287.

Zhang, X.-O., Wang, H.-B., Zhang, Y., Lu, X., Chen, L.-L., and Yang, L. 2014 Complementary sequence-mediated exon circularization. Cell 159:134-147.

Zhao, X.-Z., Wang, Y., Li, D.-X., Ren, Y.-F., and Chen, Z. 2018. Morphological characterization and phylogenetic analysis of the pathogen Phoma segeticola var. camelliae causing a new tea disease. Acta Phytopathol. Sin. 48:556-559. 